\title{
Asymptotic Analysis of Space-Time Codes in Non-Gaussian Noise and Interference
}

\author{
Ali Nezampour ${ }^{\dagger}$, Robert Schober ${ }^{\dagger}$, and Yao $\mathrm{Ma}^{\dagger \dagger}$ \\ $\dagger$ University of British Columbia. E-mail: \{alinezam, rschober\}@ece.ubc.ca \\ †† Iowa State University, E-mail: mayao@iastate.edu
}

\begin{abstract}
In this paper, we provide a framework for the asymptotic performance analysis of space-time codes (STCs) in correlated Ricean fading and non-Gaussian noise and interference. In particular, we derive a simple, asymptotically tight expression for the pairwise error probability (PEP) of coherent STCs which is valid for any type of noise and interference with finite moments. This PEP expression can be used to obtain accurate asymptotic approximations for the bit and symbol error probabilities of STCs. Our results show that while the diversity gain of an STC is independent of the type of noise, the coding gain is not and depends on certain moments of the noise and interference. We provide closed-form expressions for these moments for several practically relevant types of noise and interference. Our analytical findings are confirmed by simulations for Alamouti's ST block code and Tarokh's ST trellis code and several different types of noise and interference.
\end{abstract}

\section{INTRODUCTION}

Space-time codes (STCs) [1] are able to efficiently exploit the potential of multiple-input multiple-output (MIMO) wireless channels. Therefore, in the last few years, a tremendous amount of research has been dedicated to the design and analysis of STCs, cf. e.g. [2] and references therein. Thereby, often the asymptotic regime of high signal-to-noise ratio (SNR) is considered for both design [1] and analysis [3] since this regime leads to analytically tractable results and reveals the effects of the STC structure and the channel properties on system performance.

While the traditional analysis and design of STCs, e.g. [1][3], assumes that the only impairments of the received signal are fading and additive white Gaussian noise (AWGN), practical wireless systems also often experience non-Gaussian noise and interference ${ }^{1}$. Even if the noise is Gaussian, it may be correlated if the receive antennas are narrowly spaced [4]. Non-Gaussian impairments have been considered in [5] and [6]. In [5], STC design in the presence of co-channel interference was considered, whereas in [6], the effect of spatially dependent, temporally independent impulsive noise on STC design and analysis was studied. Both [5], [6] assume spatially independent Rayleigh fading channels.

In this paper, we present a novel, powerful, and unified framework for the calculation of the pairwise error probability (PEP) of STCs in the high SNR regime when the received signal is impaired by correlated Ricean fading and (possibly) correlated non-Gaussian noise. Thereby, we assume that the receiver has no knowledge about the statistical properties of

\footnotetext{
${ }^{1}$ To simplify our notation, in the following, "noise" refers to any additive impairment of the received signal, i.e., our definition of noise also includes what is commonly referred to as "interference".
}

the noise, and thus employs the minimum distance metric (MDM) which is optimum for AWGN. We note that, depending on the type of noise, significant performance gains are possible if the optimum maximum-likelihood (ML) metric is used instead of the MDM [6]. However, the ML metric requires knowledge of the noise distribution which may be difficult to obtain in the dynamic environments that are typical for practical wireless systems. Hence, as a pragmatic choice, receivers are usually optimized for AWGN and adopt the MDM metric. The only restriction that we impose on the noise is that all of its moments exist, which makes our results applicable to a large number of practical scenarios. The resulting PEP expression is surprisingly simple and only requires the evaluation of certain moments of the noise. Similar to the AWGN case, the novel PEP expression can be combined with the union bound to obtain tight approximations for the asymptotic bit error probability (BEP) and symbol error probability (SEP) of arbitrary space-time block codes (STBCs) and space-time trellis codes (STTCs).

The paper is organized as follows. In Section II, the considered system model is introduced. In Section III, the proposed asymptotic PEP expression is developed and some implications for STC design are discussed. The calculation of the moments of several relevant types of noise is addressed in Section IV. In Section V, some examples are given to illustrate the application of the obtained analytical results, and conclusions are drawn in Section VI.

Before we proceed, we introduce the notation and some definitions used in this paper.

Notation: In this paper, bold lower case letters $\boldsymbol{x}$ and bold upper case letters $\boldsymbol{X}$ denote vectors and matrices, respectively, and $[\boldsymbol{X}]_{i j}$ is the element of $\boldsymbol{X}$ in row $i$ and column $j$. Furthermore, $\mathcal{E}\{\cdot\}, \operatorname{Pr}\{A\},[\cdot]^{T},(\cdot)^{*},[\cdot]^{H},\|\cdot\|$, and $\operatorname{det}(\cdot)$ denote statistical expectation, the probability of event $A$, transposition, complex conjugation, Hermitian transposition, the Frobenius-norm, and the determinant of a matrix, respectively. $\otimes$ denotes the Kronecker product of two matrices and the vec operator vec $\{\boldsymbol{X}\}$ converts matrix $\boldsymbol{X}$ to a column vector by stacking the columns of $\boldsymbol{X}$ on top of each other. Furthermore, $\operatorname{diag}\left\{x_{1}, x_{2}, \ldots, x_{X}\right\}, \boldsymbol{I}_{X}$, and $\mathbf{0}_{X \times Y}$ are an $X \times X$ diagonal matrix with $x_{1}, x_{2} \ldots, x_{X}$ on its main diagonal, the $X \times X$ identity matrix, and the $X \times Y$ zero matrix, respectively. Finally, $A \doteq B$ means that $A$ is asymptotically (for high SNRs) equal to $B$ and a function $f(x)$ is $o(x)$ if $\lim _{x \rightarrow 0} f(x) / x=0$.

Moments: We define the $p$ th moment of the quadratic form $\boldsymbol{x}^{H} \boldsymbol{X} \boldsymbol{x}$ as $M_{\boldsymbol{x}}(p, \boldsymbol{X}) \triangleq \mathcal{E}\left\{\left(\boldsymbol{x}^{H} \boldsymbol{X} \boldsymbol{x}\right)^{p}\right\}$, where $\boldsymbol{X}$ and $\boldsymbol{x}$ are 
a Hermitian matrix and a complex random vector variable (RVV), respectively. If $\boldsymbol{X}$ is the $X \times X$ identity matrix, we also use the short-hand notation $M_{\boldsymbol{x}}\left(p, \boldsymbol{I}_{X}\right)=M_{\boldsymbol{x}}(p)$. We note that $M_{\boldsymbol{x}}(1)$ is the sum of the powers of the elements of $\boldsymbol{x}$.

\section{SySTEM MODEL}

We consider a MIMO system with $N_{T}$ transmit and $N_{R}$ receive antennas. The transmitter is equipped with a spacetime encoder which generates an $N \times N_{T}$ codeword matrix $\boldsymbol{C} \in \mathcal{C}$, where $N$ is the codeword (frame) length and $\mathcal{C}$ denotes the set of all codeword matrices. We assume that the $N \times N_{R}$ received signal matrix can be modeled as

$$
\boldsymbol{R}=\sqrt{\gamma} \boldsymbol{C} \boldsymbol{H}^{T}+\boldsymbol{Z},
$$

where $\boldsymbol{H}$ and $\boldsymbol{Z}$ denote the $N_{R} \times N_{T}$ channel matrix and the $N \times N_{R}$ noise matrix, respectively. We adopt the normalizations $M_{\boldsymbol{c}}(1)=N, M_{\boldsymbol{h}}(1)=N_{T} N_{R}$, and $M_{\boldsymbol{z}}(1)=N N_{R}$, where $\boldsymbol{c} \triangleq \operatorname{vec}\{\boldsymbol{C}\}, \boldsymbol{h} \triangleq \operatorname{vec}\{\boldsymbol{H}\}$, and $\boldsymbol{z} \triangleq \operatorname{vec}\left\{\boldsymbol{Z}^{T}\right\}$, i.e., $\gamma$ denotes the average SNR per receive antenna. In the following, we will discuss the properties of $\boldsymbol{H}$ and $\boldsymbol{Z}$ and the adopted decision metric more in detail.

\section{A. Channel Model}

Element $h_{n_{r} n_{t}} \triangleq[\boldsymbol{H}]_{n_{r} n_{t}}$ of channel matrix $\boldsymbol{H}$ represents the channel gain from the $n_{t}$ th transmit antenna to the $n_{r}$ th receive antenna. Eq. (1) implies that we assume that the channel matrix is constant over the duration of one codeword (quasi-static fading). Furthermore, we assume Ricean fading in this paper and $\boldsymbol{H}=\overline{\boldsymbol{H}}+\tilde{\boldsymbol{H}}$ can be split into a lineof-sight (LOS) component $\overline{\boldsymbol{H}}$ and a diffuse component $\tilde{\boldsymbol{H}}$ having zero-mean Gaussian entries $\tilde{h}_{n_{\underline{r}} n_{t}} \triangleq[\tilde{\boldsymbol{H}}]_{n_{r} n_{t}}$. The Ricean factor is defined as $K_{n_{r} n_{t}}=\left|\bar{h}_{n_{r} n_{t}}\right|^{2} / \mathcal{E}\left\{\left|\tilde{h}_{n_{r} n_{t}}\right|^{2}\right\}$, where $\bar{h}_{n_{r} n_{t}} \triangleq[\overline{\boldsymbol{H}}]_{n_{r} n_{t}}$. We note that the Ricean channel model contains the Rayleigh channel model as a special case with $K_{n_{r} n_{t}}=0, \forall n_{r}, n_{t}$. For the diffuse component $\tilde{\boldsymbol{H}}$ we adopt the popular Kronecker correlation model [7]. Therefore, the covariance matrix of $\boldsymbol{h}$ is given by

$$
\boldsymbol{R}_{h h} \triangleq \mathcal{E}\left\{\tilde{\boldsymbol{h}} \tilde{\boldsymbol{h}}^{H}\right\}=\boldsymbol{R}_{T} \otimes \boldsymbol{R}_{R}
$$

where $\tilde{\boldsymbol{h}} \triangleq \operatorname{vec}\{\tilde{\boldsymbol{H}}\}$, and $\boldsymbol{R}_{T}$ and $\boldsymbol{R}_{R}$ are the transmit-side and receive-side covariance matrices, respectively.

\section{B. Noise Model}

As usual, we assume that $\boldsymbol{Z}$ is independent from $\boldsymbol{H}$ and $\boldsymbol{C}$. However, the entries of $\boldsymbol{Z}$ may be statistically dependent, noncircularly symmetric, and non-Gaussian. The only condition that we impose on $Z$ is that all joint moments of its elements are finite. Specific examples for $\boldsymbol{Z}$ will be provided in Section IV.

\section{Minimum Distance Metric (MDM)}

We assume that the receiver uses the MDM decision rule given by

$$
\hat{\boldsymbol{C}}=\underset{\tilde{\boldsymbol{C}}}{\operatorname{argmin}}\left\{\left\|\boldsymbol{R}-\sqrt{\gamma} \tilde{\boldsymbol{C}} \boldsymbol{H}^{T}\right\|^{2}\right\}
$$

where $\hat{\boldsymbol{C}} \in \mathcal{C}$ and $\tilde{\boldsymbol{C}} \in \mathcal{C}$ denote the decoded and a hypothetical codeword matrix, respectively. The MDM is optimum if $\boldsymbol{Z}$ is AWGN [1], but is in general suboptimum for other types of noise. In the latter case, significant performance gains are possible if the ML metric is used instead of the MDM [6]. However, the application of the ML metric requires knowledge of the probability density function (pdf) of the noise, which may not be known and difficult to estimate in practice. Therefore, most practical receiver implementations use the MDM metric as a pragmatic choice even if the noise is expected to be non-Gaussian. Thus, analyzing the performance of STCs with a MDM-based receiver is of practical interest.

\section{Asymptotic Analysis of COHEREnt STCS}

In this section, we develop asymptotic expressions for the PEP of coherent STCs.

\section{A. Asymptotic Pairwise Error Probability}

The PEP is the probability that a wrong codeword $\hat{C} \in \mathcal{C}$ is decoded while $\boldsymbol{C} \in \mathcal{C}$ has been transmitted. Let $\boldsymbol{E} \triangleq \boldsymbol{C}-\hat{\boldsymbol{C}}$ denote the codeword difference matrix. Conditioned on $Z$, the PEP is a function of $\boldsymbol{E}$ and can be calculated from Eq. (3) as

$$
P_{e}(\boldsymbol{E} \mid \boldsymbol{Z})=\operatorname{Pr}\left\{\Delta<\|\boldsymbol{z}\|^{2}\right\}=\int_{0}^{\|\boldsymbol{z}\|^{2}} p_{\Delta}(x) \mathrm{d} x,
$$

where $p_{\Delta}(x)$ is the pdf of $\Delta \triangleq\|\boldsymbol{v}\|^{2}$ and $\boldsymbol{v} \triangleq$ $\operatorname{vec}\left\{\sqrt{\gamma} \boldsymbol{H} \boldsymbol{E}^{T}+\boldsymbol{Z}^{T}\right\}$ is a Gaussian RVV. Exploiting the properties of the vec operator [8], we can rewrite $\boldsymbol{v}$ as $\boldsymbol{v}=$ $\sqrt{\gamma}\left(\boldsymbol{E} \otimes \boldsymbol{I}_{N_{R}}\right) \boldsymbol{h}+\boldsymbol{z}$.

The Laplace transform $\Phi_{\Delta}(s) \triangleq \mathcal{E}\left\{e^{-s \Delta} \mid \boldsymbol{Z}\right\}$ of $p_{\Delta}(x)$ can be obtained using Turin's formula [9] as

$$
\Phi_{\Delta}(s)=\frac{\exp \left(-s \overline{\boldsymbol{v}}^{H}\left(\boldsymbol{I}_{N N_{R}}+s \boldsymbol{R}_{v v}\right)^{-1} \overline{\boldsymbol{v}}\right)}{\operatorname{det}\left(\boldsymbol{I}_{N N_{R}}+s \boldsymbol{R}_{v v}\right)},
$$

where $\overline{\boldsymbol{v}} \triangleq \sqrt{\gamma}\left(\boldsymbol{E} \otimes \boldsymbol{I}_{N_{R}}\right) \overline{\boldsymbol{h}}+\boldsymbol{z}$ and $\boldsymbol{R}_{v v} \triangleq \gamma \boldsymbol{\Gamma}$ with $\boldsymbol{\Gamma} \triangleq$ $\left(\boldsymbol{E} \otimes \boldsymbol{I}_{N_{R}}\right) \boldsymbol{R}_{h h}\left(\boldsymbol{E} \otimes \boldsymbol{I}_{N_{R}}\right)^{H}$ denote the conditional mean and covariance matrix of $\boldsymbol{v}$, respectively. Based on Eq. (2) it can be shown that the positive semi-definite matrix $\Gamma$ has $r_{t} r_{r}$ non-zero positive and $N N_{R}-r_{t} r_{r}$ zero eigenvalues, where $r_{t} \triangleq \operatorname{rank}\left\{\boldsymbol{E} \boldsymbol{R}_{T} \boldsymbol{E}^{H}\right\}$ and $r_{r} \triangleq \operatorname{rank}\left\{\boldsymbol{R}_{R}\right\}$. Consequently, $\boldsymbol{I}_{N N_{R}}+s \boldsymbol{R}_{v v}$ can be decomposed as

$$
\begin{aligned}
\boldsymbol{I}_{N N_{R}}+s \boldsymbol{R}_{v v} & =\boldsymbol{U} \boldsymbol{\Psi} \boldsymbol{U}^{H} \\
\boldsymbol{\Psi} & \triangleq\left[\begin{array}{cc}
\boldsymbol{I}_{r_{t} r_{r}}+s \gamma \boldsymbol{\Lambda} & \mathbf{0}_{r_{t} r_{r} \times\left(N N_{R}-r_{t} r_{r}\right)} \\
\mathbf{0}_{\left(N N_{R}-r_{t} r_{r}\right) \times r_{t} r_{r}} & \boldsymbol{I}_{N N_{R}-r_{t} r_{r}}
\end{array}\right]
\end{aligned}
$$

where $\boldsymbol{\Lambda} \triangleq \operatorname{diag}\left\{\lambda_{1}, \lambda_{2}, \ldots, \lambda_{r_{t} r_{r}}\right\}$ and $\boldsymbol{U}$ contain the $r_{t} r_{r}$ non-zero eigenvalues and the eigenvectors of $\Gamma$, respectively. Since $\boldsymbol{\Gamma}$ is a positive semi-definite matrix, $\boldsymbol{U}$ is a unitary matrix, i.e., $\boldsymbol{U}^{-1}=\boldsymbol{U}^{H}$. Furthermore, we decompose $\boldsymbol{U}$ into $\boldsymbol{U}=\left[\begin{array}{ll}\boldsymbol{U}_{1} & \boldsymbol{U}_{2}\end{array}\right]$, where $\boldsymbol{U}_{1}$ and $\boldsymbol{U}_{2}$ contain the $r_{t} r_{r}$ eigenvectors corresponding to the non-zero eigenvalues and the $N N_{R}-r_{t} r_{r}$ eigenvectors corresponding to the zero eigenvectors, respectively. Based on the above considerations, using 
the Neumann series [10] we can express $\left(\boldsymbol{I}_{N N_{R}}+s \boldsymbol{R}_{v v}\right)^{-1}$ as

$$
\begin{aligned}
\left(\boldsymbol{I}_{N N_{R}}+s \boldsymbol{R}_{v v}\right)^{-1}= & (\gamma s)^{-1} \boldsymbol{U}_{1} \boldsymbol{\Lambda}^{-1} \boldsymbol{U}_{1}^{H}+ \\
& \boldsymbol{U}_{2} \boldsymbol{U}_{2}^{H}+o\left((s \gamma)^{-(1+\delta)}\right) \boldsymbol{\Omega}
\end{aligned}
$$

where $\Omega$ is a finite-norm matrix, which is independent of $\gamma$, and $0<\delta<1$. Similarly, we can express the inverse determinant of $\boldsymbol{I}_{N N_{R}}+s \boldsymbol{R}_{v v}$ as

$$
\begin{aligned}
\operatorname{det}\left(\boldsymbol{I}_{N N_{R}}+s \boldsymbol{R}_{v v}\right)^{-1}=\prod_{i=1}^{r_{t} r_{r}}\left(1+s \gamma \lambda_{i}\right)^{-1} \\
=\left(\prod_{i=1}^{r_{t} r_{r}} \lambda_{i}\right)^{-1}(s \gamma)^{-r_{t} r_{r}}+o\left((s \gamma)^{-\left(r_{t} r_{r}+\delta\right)}\right) .
\end{aligned}
$$

Furthermore, using Eq. (8) and the short-hand notation $\overline{\boldsymbol{h}}_{E} \triangleq$ $\left(\boldsymbol{E} \otimes \boldsymbol{I}_{N_{R}}\right) \overline{\boldsymbol{h}}$ we can express the argument of the exponential function in Eq. (5) as $-a-s b+o\left((s \gamma)^{-\delta}\right)$ with

$$
\begin{aligned}
a & \triangleq\left(\overline{\boldsymbol{h}}_{E}+\boldsymbol{z} / \sqrt{\gamma}\right)^{H} \boldsymbol{U}_{1} \boldsymbol{\Lambda}^{-1} \boldsymbol{U}_{1}^{H}\left(\overline{\boldsymbol{h}}_{E}+\boldsymbol{z} / \sqrt{\gamma}\right) \\
b & \triangleq\left(\sqrt{\gamma} \overline{\boldsymbol{h}}_{E}+\boldsymbol{z}\right)^{H} \boldsymbol{U}_{2} \boldsymbol{U}_{2}^{H}\left(\sqrt{\gamma} \overline{\boldsymbol{h}}_{E}+\boldsymbol{z}\right) \\
& =\boldsymbol{z}^{H} \boldsymbol{U}_{2} \boldsymbol{U}_{2}^{H} \boldsymbol{z}
\end{aligned}
$$

where we have exploited the identity $\left(\boldsymbol{E} \otimes \boldsymbol{I}_{N_{R}}\right) \boldsymbol{U}_{2}=$ $\mathbf{0}_{N_{T} N_{R} \times r_{t} r_{r}}$ in Eq. (11). Combining now Eqs. (5) and (9)(11) and considering the high SNR case, we obtain for $\Phi_{\Delta}(s)$ the asymptotic expression

$$
\Phi_{\Delta}(s) \doteq \frac{\exp (-a-s b)}{\left(\prod_{i=1}^{r_{t} r_{r}} \lambda_{i}\right)(s \gamma)^{r_{t} r_{r}}} .
$$

Applying the inverse Laplace transform to $\Phi_{\Delta}(s)$ in Eq. (12) leads to

$$
p_{\Delta}(x) \doteq \frac{\exp (-a)(x-b)^{r_{t} r_{r}-1} u(x-b)}{\left(\prod_{i=1}^{r_{t} r_{r}} \lambda_{i}\right)\left(r_{t} r_{r}-1\right) ! \gamma^{r_{t} r_{r}}},
$$

where $u(x)$ denotes the unit step function. Combining Eqs. (4) and (13) yields

$$
P_{e}(\boldsymbol{E} \mid \boldsymbol{z}) \doteq \frac{\exp (-a)\left(\|\boldsymbol{z}\|^{2}-b\right)^{r_{t} r_{r}}}{\left(\prod_{i=1}^{r_{t} r_{r}} \lambda_{i}\right)\left(r_{t} r_{r}\right) ! \gamma^{r_{t} r_{r}}} .
$$

The unconditional PEP can be expressed as

$$
P_{e}(\boldsymbol{E}) \doteq \frac{\mathcal{E}\left\{\exp (-a) \boldsymbol{z}^{H} \boldsymbol{X} \boldsymbol{z}\right\}}{\left(\prod_{i=1}^{r_{t} r_{r}} \lambda_{i}\right)\left(r_{t} r_{r}\right) ! \gamma^{r_{t} r_{r}}},
$$

where we have the definition

$$
\boldsymbol{X} \triangleq \boldsymbol{I}_{N N_{R}}-\boldsymbol{U}_{2} \boldsymbol{U}_{2}^{H}=\boldsymbol{U}_{1} \boldsymbol{U}_{1}^{H} .
$$

Using the expansion $\exp (x)=\sum_{k=0}^{\infty} x^{k} / k$ ! and Eq. (10), we can rewrite the exponential function in Eq. (15) as

$$
\exp (-a)=\exp \left(-\overline{\boldsymbol{h}}_{E}^{H} \boldsymbol{U}_{1} \boldsymbol{\Lambda}^{-1} \boldsymbol{U}_{1}^{H} \overline{\boldsymbol{h}}_{E}\right)(1+f(\boldsymbol{z}) / \sqrt{\gamma}),
$$

where $f(\boldsymbol{z})$ can be written as a sum of products of the form $C_{\kappa_{1}, \nu_{1}, \cdots, \kappa_{N N_{R}}, \nu_{N N_{R}}} z_{1}^{\kappa_{1}}\left(z_{1}^{*}\right)^{\nu_{1}} \cdots z_{N N_{R}}^{\kappa_{N N_{R}}}\left(z_{N N_{R}}^{*}\right)^{\nu_{N N_{R}}}$, where $C_{\kappa_{1}, \nu_{1}, \cdots, \kappa_{N N_{R}}, \nu_{N N_{R}}}$ are coefficients that are non-increasing in $\gamma, z_{l}, 1 \leq l \leq N N_{R}$, denote the elements of $\boldsymbol{z}$, and $\kappa_{l} \geq 0$ and $\nu_{l} \geq 0$ are integers. Assuming now that all moments of $z$ exist (i.e., $\mathcal{E}\left\{\boldsymbol{z}^{H} \boldsymbol{X} \boldsymbol{z} f(\boldsymbol{z})\right\}<\infty$ ), for $\gamma \rightarrow \infty$, we obtain for the PEP the simple asymptotic expression

$$
P_{e}(\boldsymbol{E}) \doteq \frac{\alpha M_{\boldsymbol{z}}\left(r_{t} r_{r}, \boldsymbol{X}\right)}{\gamma^{r_{t} r_{r}}}=\frac{\alpha M_{\boldsymbol{y}}\left(r_{t} r_{r}\right)}{\gamma^{r_{t} r_{r}}}
$$

with

$$
\alpha \triangleq \frac{\exp \left(-\overline{\boldsymbol{h}}_{E}^{H} \boldsymbol{U}_{1} \boldsymbol{\Lambda}^{-1} \boldsymbol{U}_{1}^{H} \overline{\boldsymbol{h}}_{E}\right)}{\left(r_{t} r_{r}\right) ! \prod_{i=1}^{r_{t} r_{r}} \lambda_{i}},
$$

where we used the definition $\boldsymbol{y} \triangleq \boldsymbol{U}_{1}^{H} \boldsymbol{z}$. Eq. (18) can be viewed as a generalization of existing asymptotic results for AWGN in e.g. [3] to the non-Gaussian case. We note from Eq. (18) that the type of noise impacts the asymptotic PEP only via the moment $M_{\boldsymbol{z}}\left(r_{t} r_{r}, \boldsymbol{X}\right)$. Furthermore, for $r_{t}$ and $r_{r}$ the inequalities $1 \leq r_{t} \leq \min \left\{\operatorname{rank}\{\boldsymbol{E}\}, \operatorname{rank}\left\{\boldsymbol{R}_{T}\right\}\right\} \leq$ $\min \left\{N, N_{T}\right\}$ and $1 \leq r_{r} \leq N_{R}$ hold. For the special case of full-rank square STBCs with $N=N_{T}$ and full-rank transmit and receive covariance matrices, we obtain $r_{t}=N_{T}, r_{r}=$ $N_{R}$, and $\boldsymbol{U}=\boldsymbol{U}_{1}$. Therefore, in this case, $\boldsymbol{X}=\boldsymbol{I}_{N_{T} N_{R}}$ and Eqs. (18) and (19) can be simplified to

$$
P_{e}(\boldsymbol{E}) \doteq \frac{\alpha M_{\boldsymbol{z}}\left(N_{T} N_{R}\right)}{\gamma^{N_{T} N_{R}}},
$$

with

$$
\alpha \triangleq \frac{\exp \left(-\overline{\boldsymbol{h}}^{H}\left(\boldsymbol{R}_{T}^{-1} \otimes \boldsymbol{R}_{R}^{-1}\right) \overline{\boldsymbol{h}}\right)}{\left(N_{T} N_{R}\right) !\left[\operatorname{det}\left(\boldsymbol{E} \boldsymbol{E}^{H}\right) \operatorname{det}\left(\boldsymbol{R}_{T}\right)\right]^{N_{R}}\left[\operatorname{det}\left(\boldsymbol{R}_{R}\right)\right]^{N_{T}}} .
$$

Therefore, for full-rank square STBCs (such as e.g. Alamouti's code) the code does not affect the way in which the noise statistics impact the PEP because $M_{\boldsymbol{z}}\left(N_{T} N_{R}\right)$ is independent of the code. In contrast, for codes with $N>N_{T}$ (e.g. STTCs) this is not the case since $M_{\boldsymbol{z}}\left(r_{t} r_{r}, \boldsymbol{X}\right)$ also depends on the code via $\boldsymbol{U}_{1}$, cf. Eq. (16). If we specialize Eqs. (20) and (21) to $N_{T}=1$, as expected we obtain [11, Eqs. (11), (12)], which were derived for single-antenna transmission and maximumratio combining (MRC).

\section{B. Implications for STC Design}

For STC design it is convenient to express the asymptotic PEP as $P_{e}(\boldsymbol{E}) \doteq\left(G_{c} \gamma\right)^{-G_{d}}$ [1], [12], where $G_{c}$ and $G_{d}$ are referred to as the coding gain and the diversity gain, respectively. Considering Eqs. (18) and (19), we obtain

$$
\begin{aligned}
G_{d} & =r_{t} r_{r} \\
G_{c}[\mathrm{~dB}] & =\frac{10}{r_{t} r_{r}} \log _{10}(\alpha)+\frac{10}{r_{t} r_{r}} \log _{10}\left(M_{\boldsymbol{y}}\left(r_{t} r_{r}\right)\right)
\end{aligned}
$$

Since on a double logarithmic scale $G_{d}$ determines the slope of the PEP curve, the primary goal of most STC designs is to maximize the diversity gain by maximizing $r_{t}$ [1]. Since $G_{d}$ is independent of the type of noise, this rank criterion is also independent of the type of noise in the system. Therefore, our findings here generalize the results of [1] and [6] which show the importance of the rank criterion for AWGN and impulsive noise, respectively.

The secondary STC design goal is usually the maximization of the coding gain [1]. Eq. (23) shows that the coding gain 
consists of two parts. The first term on the right hand side (RHS) of Eq. (23) is independent of the type of noise, whereas the second term potentially depends on the type of noise via $M_{\boldsymbol{y}}\left(r_{t} r_{r}\right)$. For AWGN $M_{\boldsymbol{y}}\left(r_{t} r_{r}\right)$ is independent of $\boldsymbol{X}$ as will be shown in Section IV, and for full-rank square STBCs $M_{\boldsymbol{y}}\left(r_{t} r_{r}\right)=M_{\boldsymbol{z}}\left(N_{T} N_{R}\right)$ holds for all types of noise. Therefore, in these two special cases the second term on the RHS of Eq. (23) can be ignored for STC optimization. This means that full-rank square STBCs optimized for AWGN are also optimum for any other type of noise. In contrast, codes with $N>N_{T}$ optimized for AWGN are not necessarily optimum for other types of noise. If $M_{\boldsymbol{y}}\left(r_{t} r_{r}\right)$ is known at the receiver, the expression in Eq. (23) can be used for code search. However, this aspect is beyond the scope of this paper and will be explored in future work.

\section{Bit and Symbol Error Probability}

Based on the PEP an upper bound on the BEP can be obtained via the union bound [13]

$$
\mathrm{BEP} \leq \frac{1}{n_{c}} \sum_{\boldsymbol{C}} P(\boldsymbol{C}) \sum_{\boldsymbol{C} \neq \hat{\boldsymbol{C}}} n(\boldsymbol{C}, \hat{\boldsymbol{C}}) P_{e}(\boldsymbol{C}-\hat{\boldsymbol{C}}),
$$

where $n_{c}, P(\boldsymbol{C})$, and $n(\boldsymbol{C}, \hat{\boldsymbol{C}})$ denote the number of information bits per codeword, the probability that $C$ is transmitted, and the number of bit errors if $C \in \mathcal{C}$ is transmitted but $\hat{\boldsymbol{C}} \in \mathcal{C}$ is detected, respectively. Typically, a tight asymptotic upper bound can be obtained by including only a few dominant terms in Eq. (24). For most known codes the number of terms required to obtain an upper bound is well documented in the literature and depends on the structure of the code, the modulation scheme, and the diversity order, e.g. [13], [14]. We found the number of terms required to obtain a tight asymptotic upper bound of the BEP to be independent of the type of noise. We note that depending on the structure of the STC (e.g. orthogonal STBC), Eq. (24) can be simplified significantly [2], [13]. Furthermore, if the bits are mapped to $M$-ary scalar symbols $s_{i}, 1 \leq i \leq Q$, which are subsequently mapped to STC matrices $\boldsymbol{C}$, a tight asymptotic upper bound on the SEP of the $s_{i}$ can be obtained with a formula similar to Eq. (24). Because of space limitation we omit further details here and refer the interested reader to [2], [13].

\section{Calculation of the Noise Moments}

The general PEP expression in Eq. (18) requires the calculation of the moments $M_{\boldsymbol{y}}\left(r_{t} r_{r}\right)=M_{\boldsymbol{z}}\left(r_{t} r_{r}, \boldsymbol{X}\right)$. Using $x_{i j} \triangleq[\boldsymbol{X}]_{i j}, s \triangleq N N_{R}$, and the multinomial expansion, we obtain

$$
\begin{aligned}
& M_{\boldsymbol{y}}\left(r_{t} r_{r}\right)= \sum_{\substack{k_{1}+\ldots+k_{s}=r_{t} r_{r}\\
}}\left(\begin{array}{c}
n \\
k_{1}, \ldots, k_{s}
\end{array}\right) x_{11}^{k_{1}} x_{12}^{k_{2}} \ldots x_{s s}^{k_{s}} \\
& \cdot \mathcal{E}\left\{\left(z_{1} z_{1}^{*}\right)^{k_{1}}\left(z_{1} z_{2}^{*}\right)^{k_{2}} \ldots\left(z_{s} z_{s}^{*}\right)^{k_{s}}\right\} .
\end{aligned}
$$

Therefore, calculation of $M_{\boldsymbol{y}}\left(r_{t} r_{r}\right)$ requires the evaluation of the joint moments $\mathcal{E}\left\{\left(z_{1} z_{1}^{*}\right)^{k_{1}}\left(z_{1} z_{2}^{*}\right)^{k_{2}} \ldots\left(z_{s} z_{s}^{*}\right)^{k_{s}}\right\}$. While Eq. (25) is very general and can always be applied, simpler closed-form expressions can be obtained for specific types of noise. Some examples are discussed in the following.

\section{A. Additive White Gaussian Noise}

It is instructive to consider the AWGN case. If $z$ is an AWGN vector, $\boldsymbol{y}=\boldsymbol{U}_{1}^{H} \boldsymbol{z}$ is also an AWGN vector of length $r_{t} r_{r}$ since the columns of $\boldsymbol{U}_{1}$ are orthogonal. The elements of $\boldsymbol{y}$ have variance $\sigma_{y}^{2}=1$. Therefore, we obtain for $M_{\boldsymbol{y}}\left(r_{t} r_{r}\right)$ the expression provided in Table I. As already mentioned in Section III-B, for AWGN $M_{\boldsymbol{y}}\left(r_{t} r_{r}\right)$ is independent of $\boldsymbol{X}$ and the provided PEP expressions can be simplified to expressions known from the literature. For example, it can be verified that for full-rank $\boldsymbol{E}$, full-rank $\boldsymbol{R}_{T}$ and $\boldsymbol{R}_{R}$, Rayleigh fading, and AWGN, Eqs. (18) and (19) are equivalent to the asymptotic PEP expression in [3, Proposition 3].

\section{B. Correlated Gaussian Noise (GN)}

Zero-mean correlated GN may be caused either by interference or correlated thermal noise in case of narrowly spaced receive antennas [4]. Denote the correlation matrix of $z$ by $\boldsymbol{R}_{z z} \triangleq \mathcal{E}\left\{\boldsymbol{z} \boldsymbol{z}^{H}\right\}$. If spatial and temporal noise correlation are independent, $\boldsymbol{R}_{z z}=\boldsymbol{R}_{z z, t} \otimes \boldsymbol{R}_{z z, s}$, where $\boldsymbol{R}_{z z, t}$ and $\boldsymbol{R}_{z z, s}$ are the $N \times N$ temporal and the $N_{R} \times N_{R}$ spatial correlation matrices, respectively. If the noise is temporally uncorrelated, $\boldsymbol{R}_{z z, t}=\boldsymbol{I}_{N}$. The correlation matrix of $\boldsymbol{y}$ is given by $\boldsymbol{R}_{y y}=\boldsymbol{U}_{1}^{H} \boldsymbol{R}_{z z} \boldsymbol{U}_{1}$. Therefore, after tedious but straightforward calculations we obtain for $M_{\boldsymbol{y}}\left(r_{t} r_{r}\right)$ the expression given in Table I, where $\eta_{i}, 1 \leq i \leq r_{t} r_{r}$ denote the eigenvalues of $\boldsymbol{R}_{y y}$.

\section{Gaussian Mixture Noise (GMN)}

In general, we may distinguish between spatially independent and spatially dependent GMN, cf. [6], [15].

1) Spatially Independent GMN: The pdf of spatially and temporally independent and identically distributed (i.i.d.) GMN is given by

$$
p_{z}\left(z_{n_{r}}[n]\right)=\sum_{i=1}^{I} \frac{c_{i}}{\pi \sigma_{i}^{2}} \exp \left(-\frac{\left|z_{n_{r}}[n]\right|^{2}}{\sigma_{i}^{2}}\right),
$$

where $c_{i}>0, \sum_{i=1}^{I} c_{i}=1$, and $\sigma_{i}^{2}, 1 \leq i \leq I$, are constants. Here, $z_{n_{r}}[n]$ denotes the noise at receive antenna $n_{r}, 1 \leq n_{r} \leq$ $N_{R}$, at time $n, 0 \leq n<N$. Conditioned on state $i, 1 \leq i \leq I$, $z_{n_{r}}[n]$ is a Gaussian random variable $(\mathrm{RV})$ with variance $\sigma_{i}^{2}$. Since vector $\boldsymbol{z} \triangleq\left[z_{1}[0] z_{2}[0] \ldots z_{N_{R}}[0] z_{1}[1] \ldots z_{N_{R}}[N-\right.$ 1]] ${ }^{T}$ has $N N_{R}$ independent elements and each element can have $I$ different variances, there are $I^{N N_{R}}$ different possible states for $\boldsymbol{z}$. Conditioned on state $i, 1 \leq i \leq I^{N N_{R}}, \boldsymbol{z}$ is a Gaussian RVV with diagonal covariance matrix $\boldsymbol{\Sigma}_{i} \triangleq$ $\mathcal{E}\left\{\boldsymbol{z} \boldsymbol{z}^{H}\right\}$. The $N N_{R}$ main diagonal elements of the set of covariance matrices $\boldsymbol{\Sigma}_{i}, 1 \leq i \leq I^{N N_{R}}$, contain all $I^{N N_{R}}$ possible different combinations of variances $\sigma_{i}^{2}, 1 \leq i \leq I$. Consequently, conditioned on state $i$, vector $\boldsymbol{y}$ is also a Gaussian RVV with $r_{t} r_{r} \times r_{t} r_{r}$ covariance matrix $\boldsymbol{R}_{i}=\boldsymbol{U}_{1}^{H} \boldsymbol{\Sigma}_{i} \boldsymbol{U}_{1}$, and the pdf of $\boldsymbol{y}$ can be written as

$$
p_{\boldsymbol{y}}(\boldsymbol{y})=\sum_{i=1}^{I^{N N_{R}}} \frac{\alpha_{i}}{\pi^{n_{t} n_{r}} \operatorname{det}\left(\boldsymbol{R}_{i}\right)} \exp \left(-\boldsymbol{y}^{H} \boldsymbol{R}_{i}^{-1} \boldsymbol{y}\right)
$$


where $\alpha_{i}=\prod_{m=1}^{I} c_{m}^{k_{m}}$ and $k_{m}$ is the number of times $\sigma_{m}^{2}$ is contained in $\boldsymbol{\Sigma}_{i}$, i.e., $\sum_{m=1}^{I} k_{m}=N N_{R}$ holds. Based on Eq. (27) the moment $M_{\boldsymbol{y}}\left(r_{t} r_{r}\right)$ can be calculated as given in Table I, where $\eta_{m}[i], 1 \leq m \leq r_{t} r_{r}$, denotes the eigenvalues of $\boldsymbol{R}_{i}$. We note that the outer sum in the expression for $M_{\boldsymbol{y}}\left(r_{t} r_{r}\right)$ in Table I is over $I^{N N_{R}}$ terms and $I^{N N_{R}}$ is potentially a very large number. However, in practice, so-called $\epsilon$-mixture noise with $I=2$ is usually considered and relatively small values for $N$ suffice to capture all relevant (dominant) error events in the union bound in Eq. (24). For $\epsilon$-mixture noise with variance $\sigma_{z}^{2}, c_{1}=1-\epsilon, c_{2}=\epsilon$, $\sigma_{1}^{2}=\sigma_{z}^{2} /(1-\epsilon+\kappa \epsilon)$, and $\sigma_{2}^{2}=\kappa \sigma_{1}^{2}$ with $0<\epsilon<1$ and $\kappa>1$.

2) Spatially Dependent GMN: The pdf of spatially dependent but temporally i.i.d. GMN is given by [6]

$$
p_{z}(\boldsymbol{z}[n])=\sum_{i=1}^{I} \frac{c_{i}}{\pi^{N_{R}} \sigma_{i}^{2 N_{R}}} \exp \left(-\frac{|\boldsymbol{z}[n]|^{2}}{\sigma_{i}^{2}}\right),
$$

where $\boldsymbol{z}[n] \triangleq\left[z_{1}[n] \ldots z_{N_{R}}[n]\right]^{T}$ contains the noise variables of all receive antennas at time $n, 0 \leq n<N$, and $c_{i}>0$, $\sum_{i=1}^{I} c_{i}=1$, and $\sigma_{i}^{2}, 1 \leq i \leq I$, are again constants. Spatially dependent GMN can be treated in a similar way as spatially independent GMN. The main difference is that now there are only $I^{N}$ different correlation matrices $\boldsymbol{\Sigma}_{i}, 1 \leq i \leq I^{N}$, for $\boldsymbol{z}$ since all $z_{n_{r}}[n]$ belonging to the same time instant $n, 0 \leq$ $n<N$, have the same variance. Therefore, the pdf of $\boldsymbol{y}$ is still given by Eq. (27) if we replace $I^{N N_{R}}$ by $I^{N} \cdot \alpha_{i}$ is still given by $\alpha_{i}=\prod_{m=1}^{I} c_{m}^{k_{m}}$ but now $k_{m}$ is the number of times $\sigma_{m}^{2}$ is contained in $\Sigma_{i}$ divided by $N_{R}$, i.e., $\sum_{m=1}^{I} k_{m}=N$ holds. The moment $M_{\boldsymbol{y}}\left(r_{t} r_{r}\right)$ for spatially dependent GMN is also given in Table $\mathrm{I}$.

\section{Asynchronous Co-channel Interference (CCI)}

Rayleigh-faded asynchronous CCI can be modeled as

$$
z_{n_{r}}[n]=g_{n_{r}} \sum_{k=-k_{l}}^{k_{u}} f[k] b[n-k], \quad 1 \leq n_{r} \leq N_{R},
$$

where $f[k]\left(-k_{l} \leq k \leq k_{u}, k_{l}, k_{u} \geq 0\right), b[k] \in \mathcal{B}$, and $g_{n_{r}}$ denote the effective CCI impulse response, the i.i.d. CCI symbols belonging to an $M$-ary symbol alphabet $\mathcal{B}$, and the CCI fading gain, respectively. The CCI fading gains of different antennas are (possibly correlated) zero-mean Gaussian RVs and we denote the CCI covariance matrix as $\boldsymbol{R}_{g g} \triangleq \mathcal{E}\left\{\boldsymbol{g g}^{H}\right\}$, where $\boldsymbol{g} \triangleq\left[g_{1} \ldots g_{N_{R}}\right]^{T}$. The CCI impulse response is given by $f[k] \triangleq f(k T+\tau)$, where $f(t), T$, and $\tau$ denote the convolution of the transmit pulse shape of the interferer and the receiver input filter impulse response of the user, the symbol duration of the user, and the delay between the interferer and the user, respectively.

Based on Eq. (29) the noise vector $z$ can be represented as

$$
\boldsymbol{z}=(\boldsymbol{F} \boldsymbol{b}) \otimes \boldsymbol{g},
$$

where the $i$ th row of the $N \times L_{b}$ matrix $\boldsymbol{F}$ is given by $\left[\mathbf{0}_{1 \times(i-1)} f\left[-k_{l}\right] f\left[-k_{l}+1\right] \ldots f\left[k_{u}\right] \mathbf{0}_{1 \times(N-i)}\right], 1 \leq i \leq N$, and CCI vector $\boldsymbol{b} \triangleq\left[b\left[-k_{u}\right] b\left[-k_{u}+1\right] \ldots b\left[N-1+k_{l}\right]\right]^{T}$ has
TABLE I

MOMENTS $M_{\boldsymbol{y}}(p) \triangleq \mathcal{E}\left\{\left(\boldsymbol{y}^{H} \boldsymbol{y}\right)^{p}\right\}$ OF SOME RELEVANT TYPES OF NOISE

\begin{tabular}{|c|c|}
\hline Model for $\boldsymbol{z}$ & Moments $M_{\boldsymbol{y}}(p)$ \\
\hline AWGN & $\frac{(2 p-1) !}{(p-1) !} \sigma_{y}^{2 p}$ \\
\hline Correlated GN & $p ! \sum_{k_{1}+\ldots+k_{p}=p} \eta_{1}^{k_{1}} \cdot \ldots \cdot \eta_{p}^{k_{p}}$ \\
\hline Spat. Ind. GMN & $p ! \sum_{i=1}^{I^{N N_{R}}} \alpha_{i} \sum_{k_{1}+\ldots+k_{p}=p} \eta_{1}^{k_{1}}[i] \cdot \ldots \cdot \eta_{p}^{k_{p}}[i]$ \\
\hline Spat. Dep. GMN & $p ! \sum_{i=1}^{I^{N}} \alpha_{i} \sum_{k_{1}+\ldots+k_{p}=p} \eta_{1}^{k_{1}[i] \cdot \ldots \cdot \eta_{p}^{k_{p}}[i]}$ \\
\hline Asynch. CCI & $\frac{p !}{M^{L_{b}}} \sum_{\boldsymbol{b} \in \mathcal{B}^{L_{b}}} \sum_{k_{1}+\ldots+k_{p}=p} \eta_{1}^{k_{1}}(\boldsymbol{b}) \cdot \ldots \cdot \eta_{p}^{k_{p}}(\boldsymbol{b})$ \\
\hline
\end{tabular}
$\boldsymbol{z}$, WHERE $\boldsymbol{y}=\boldsymbol{U}_{1}^{H} \boldsymbol{z}$ AND $p \triangleq r_{t} r_{r}$. ALL VARIABLES IN THIS TABLE ARE DEFINED IN SECTION IV.

length $L_{b} \triangleq N+k_{u}+k_{l}$. Conditioned on $\boldsymbol{b}, \boldsymbol{y}=\boldsymbol{U}_{1}^{H}[(\boldsymbol{F} \boldsymbol{b}) \otimes \boldsymbol{g}]$ is a zero-mean Gaussian RVV with $r_{t} r_{r} \times r_{t} r_{r}$ covariance matrix

$$
\boldsymbol{R}_{y y}(\boldsymbol{b}) \triangleq \mathcal{E}\left\{\boldsymbol{y} \boldsymbol{y}^{H} \mid \boldsymbol{b}\right\}=\boldsymbol{U}_{1}^{H}\left[\left(\boldsymbol{F} \boldsymbol{b} \boldsymbol{b}^{H} \boldsymbol{F}^{H}\right) \otimes \boldsymbol{R}_{g g}\right] \boldsymbol{U}_{1},
$$

where we have exploited some properties of the Kronecker product, cf. e.g. [8], to arrive at Eq. (31). Consequently, the moment $M_{\boldsymbol{y}}\left(r_{t} r_{r}\right)$ for asynchronous CCI can be obtained as given in Table I, where $\eta_{m}(\boldsymbol{b}), 1 \leq m \leq r_{t} r_{r}$, denotes the eigenvalues of $\boldsymbol{R}_{y y}(\boldsymbol{b})$. The outer sum in the expression for $M_{\boldsymbol{y}}\left(r_{t} r_{r}\right)$ in Table I is over all $M^{L_{b}}$ possible vectors $\boldsymbol{b}$. However, since typically $N \leq 10$ suffices to account for the dominant error events of practical STCs, the computation of $M_{\boldsymbol{y}}\left(r_{t} r_{r}\right)$ is quite fast. Also note that $M_{\boldsymbol{y}}\left(r_{t} r_{r}\right)$ has to be calculated only once as it does not depend on the SNR.

\section{NUMERICAL RESULTS AND DiSCUSSIONS}

In this section, we verify the derived analytical expressions for some practically relevant cases with computer simulations. For this purpose, we combine the asymptotic PEP with the truncated union bound to arrive at asymptotic upper bounds for the BEP and SEP as discussed in Section III-C. The required noise moments were obtained with the expressions given in Table I. In all figures, we show the BEP as functions of symbol SNR per receive antenna ${ }^{2}$.

Fig. 1 shows the SEP of Alamouti's STBC $(N=2)$ with $N_{T}=2$ transmit antennas and both $N_{R}=1$ and $N_{R}=2$ receive antennas. The STC matrices are constructed from $8-$ ary phase-shift keying (8-PSK) symbols and transmission over an i.i.d. Ricean fading channel $(K=0 \mathrm{~dB})$ is assumed. The performance of the STC for AWGN, 8-PSK asynchronous CCI, and spatially dependent $\epsilon$-mixture noise with two different sets of parameters $(\epsilon=0.25, \kappa=10$ and $\epsilon=0.10, \kappa=$ 10) are compared. For the CCI example, we assume that both transmitter and receiver use square-root raised cosine filters with roll-off factor 0.22 , a delay of $\tau=T / 4$ for the interferer,

\footnotetext{
${ }^{2}$ Since, in this paper, we refer to any additive impairment as "noise", we use the term "SNR" even if the received signal is only impaired by what is traditionally referred to as "interference".
} 


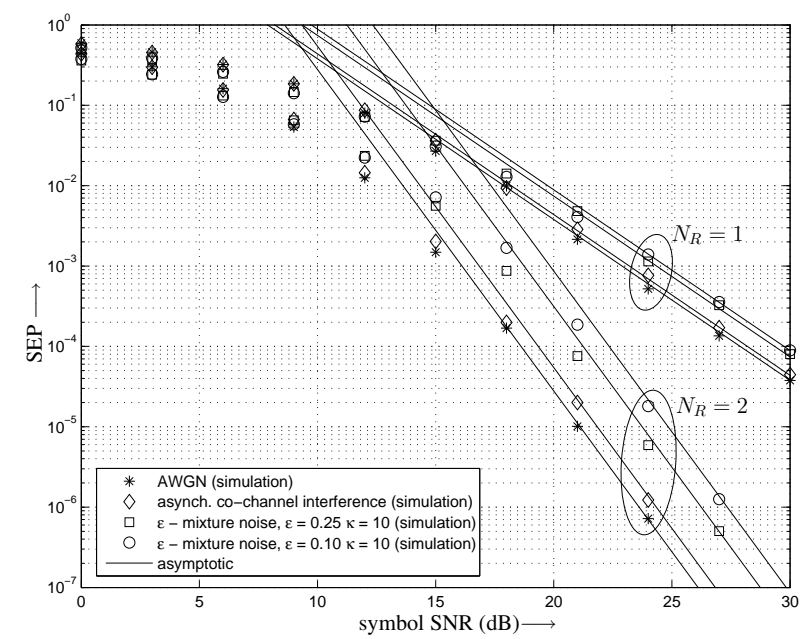

Fig. 1. SEP of Alamouti's STBC $\left(N=N_{T}=2\right)$ for different types of noise. 8-PSK modulation, i.i.d. Ricean fading ( $K=0 \mathrm{~dB}), N_{R}=1$ and $N_{R}=2$. Markers: Simulated SEP. Lines: Asymptotic SEP.

and, in case of $N_{R}=2$, uncorrelated CCI fading gains. We observe from Fig. 1 that for all considered types of noise the simulation points quickly approach the asymptotic curves as the SNR increases. Furthermore, the considered non-Gaussian noises degrate performance compared to AWGN, which, in part, can be attributed to the fact that the MDM is suboptimum in these cases. We also note that the performance loss caused by the non-Gaussian noise increases with the diversity order.

In Fig. 2, we have simulated the BEP of the 4-state STTC in [1] with $N_{T}=2$ and 4-PSK modulation over a Rayleigh fading channel impaired by Gaussian noise. For $N_{R}=2$, we assume that both the fading and the noise are spatially correlated. For the fading we assume $\boldsymbol{R}_{T}=\boldsymbol{I}_{N_{T}}$ and $\boldsymbol{R}_{R}$ is a Toeplitz matrix with first row $\left[\begin{array}{ll}1 & \rho_{h}\end{array}\right]$. Similarly, we assume that the noise is temporally independent but has a spatial covariance matrix $\boldsymbol{R}_{z z, s}$ with first row $\left[1 \rho_{z}\right]$. Fig. 2 includes BEP curves for all possible combinations of $\rho_{h}=\{0.0,0.7\}$ and $\rho_{z}=\{0.0,0.7\}$. We considered error events of up to lengths two and three in Eq. (24) to obtain a tight asymptotic upper bound for $N_{R}=1$ and $N_{R}=2$, respectively. We observe from Fig. 2 that not only the fading correlation, but also the noise correlation negatively affects the performance of STCs. However, the STC is more sensitive to fading correlation than to noise correlation.

\section{CONCLUSION}

In this paper, we have presented simple and insightful asymptotic PEP expressions for STCs operating in correlated Ricean fading and non-Gaussian noise and interference. The presented results are very general and are applicable to many practical scenarios since they are valid for any coherent STC and the only mild condition that we require is that all moments of the noise and the interference are finite. Our results show that the diversity gain of a STC is independent of the type of noise, whereas the coding gain is affected by the properties of the noise. Furthermore, for full-rank square STBCs the code design has no influence on how the type of noise affects the

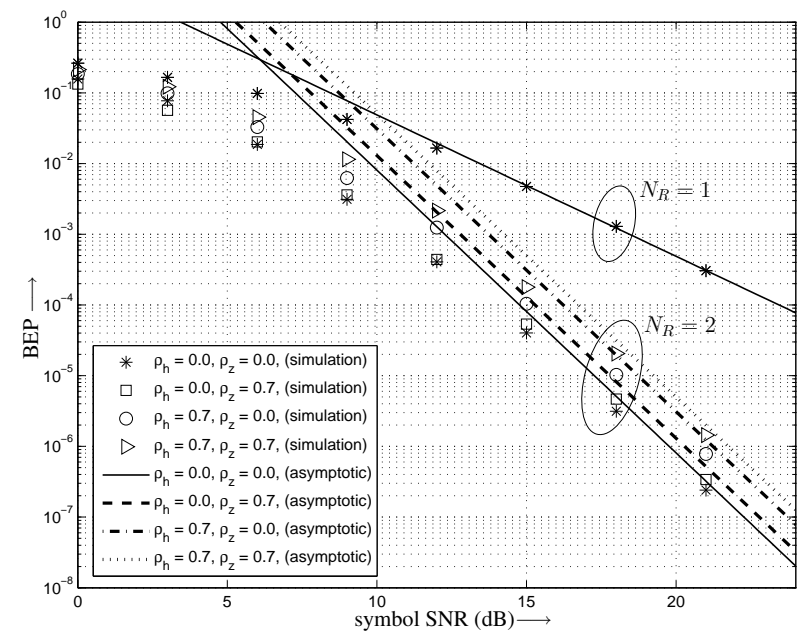

Fig. 2. BEP of 4-state STTC $\left(N_{T}=2\right)$ for i.i.d. and correlated Rayleigh fading and i.i.d. and correlated Gaussian noise. 4-PSK modulation, $N_{R}=1$ and $N_{R}=2$. Markers: Simulated BEP. Lines: Asymptotic BEP.

coding gain. In contrast, for non-square STBCs and STTCs the code design does have an impact on how the coding gain is affected by the type of noise. This dependency may be explored more in detail in future research and could give raise to new STC designs.

\section{REFERENCES}

[1] V. Tarokh, N. Seshadri, and A.R. Calderbank. Space-Time Codes for High Data Rate Wireless Communication: Performance Criterion and Code Construction. IEEE Trans. Inform. Theory, 44:744-765, March 1998.

[2] H. Jafarkhani. Space-Time Coding, Theory and Practice. Cambridge University Press, Cambridge, UK, 2005.

[3] M. Brehler and M. K. Varanasi. Asymptotic Error Probability Analysis of Quadratic Receivers in Rayleigh-Fading Channels with Applications to a Unified Analysis of Coherent and Noncoherent Space-Time Receivers. IEEE Trans. Inform. Theory, 47:2383-2399, September 2001.

[4] S. Krusevac, P. Rapajic, and R. Kennedy. Channel Capacity Estimation for MIMO Systems with Correlated Noise. In Proceedings of the Global Telecommun. Conf. (Gobecom), pages 2812-2816, St. Louis, MO, 2005.

[5] A. Arunachalam and H. El Gamal. Space-Time Coding for MIMO Systems with Co-channel Interference. IEEE Trans. on Wireless Commun., 3(6):1953-1958, November 2004

[6] P. Gao and C. Tepedelenlioglu. Space-Time Coding over Fading Channels with Impulsive Noise. IEEE Trans. on Wireless Commun., 6:220-229, January 2007

[7] J. P. Kermoal, L. Schumacher, K. I. Pedersen, P. E. Mogensen, and F. Frederiksen. A Stochastic MIMO Radio Channel Model with Experimental Validation. IEEE J. Select. Areas Commun., 20:12111226, August 2002.

[8] T.K. Moon and W.C. Stirling. Mathematical Methods and Algorithms for Signal Processing. Prentice Hall, New York, 2000.

[9] G. Turin. The characteristic function of hermetian quadratic forms in complex normal random variables. Biometrika, pages 199-201, June 1960.

[10] C. Meyer. Matrix Analysis and Applied Linear Algebra. SIAM, Philadelphia, 2000.

[11] A. Nezampour, A. Nasri, R. Schober, and Y. Ma. Asymptotic BEP and SEP of MRC in Correlated Ricean Fading and Non-Gaussian Noise. IEEE Vehicular Technology Conference (VTC), Baltimore, October 2007.

[12] J.G. Proakis. Digital Communications. McGraw-Hill, New York, forth edition, 2001.

[13] M.K. Simon and M.-S. Alouini. Digital Communication over Fading Channels. Wiley, Hoboken, New Jersey, 2005.

[14] A. Hedayat, H. Shah, and A. Nosratinia. Analysis of Sace-Time Coding in Correlated Fading Channels. IEEE Trans. on Wireless Commun., 4:2882-2891, November 2005.

[15] C. Tepedelenlioglu and P. Gao. On Diversity Reception Over Fading Channels with Impulsive Noise. IEEE Trans. Veh. Technol., 54:20372047, November 2005. 